\title{
Belphégor
}

Littérature populaire et culture médiatique

17 | 2019

Mutations des légitimités dans les productions culturelles contemporaines

\section{Diana Holmes, Middlebrow Matters. Women's reading and the literary canon in France since the Belle Époque} Jan Baetens

\section{CpenEdition}

\section{Journals}

Electronic version

URL: https://journals.openedition.org/belphegor/1458

DOI: $10.4000 /$ belphegor.1458

ISSN: 1499-7185

Publisher

LPCM

Electronic reference

Jan Baetens, "Diana Holmes, MIDDlebrow MATTERS. WOMEN'S READINg AND THE LITERARY CANON IN frANCE SINCE THE beLLE ÉPOQUE", Belphégor [Online], 17 | 2019, Online since 16 April 2019, connection on 25 August 2021. URL: http://journals.openedition.org/belphegor/1458 ; DOI: https://doi.org/ 10.4000/belphegor.1458

This text was automatically generated on 25 August 2021.

\section{(1) $\$$}

Belphégor est mis à disposition selon les termes de la Licence Creative Commons Attribution - Pas d'Utilisation Commerciale - Pas de Modification 4.0 International. 
Diana Holmes, Middlebrow Matters. Women's reading and the literary canon in France since the Belle Époque

Jan Baetens

\section{REFERENCES}

Diana Holmes, Middlebrow Matters. Women's reading and the literary canon in France since the Belle Époque, Contemporary French and Francophone Cultures, 57, Liverpool: Liverpool University Press, 256 p., £30.00 ISBN: 978-1-786-94156-5 (relié), 9781786949523 (eBook) 


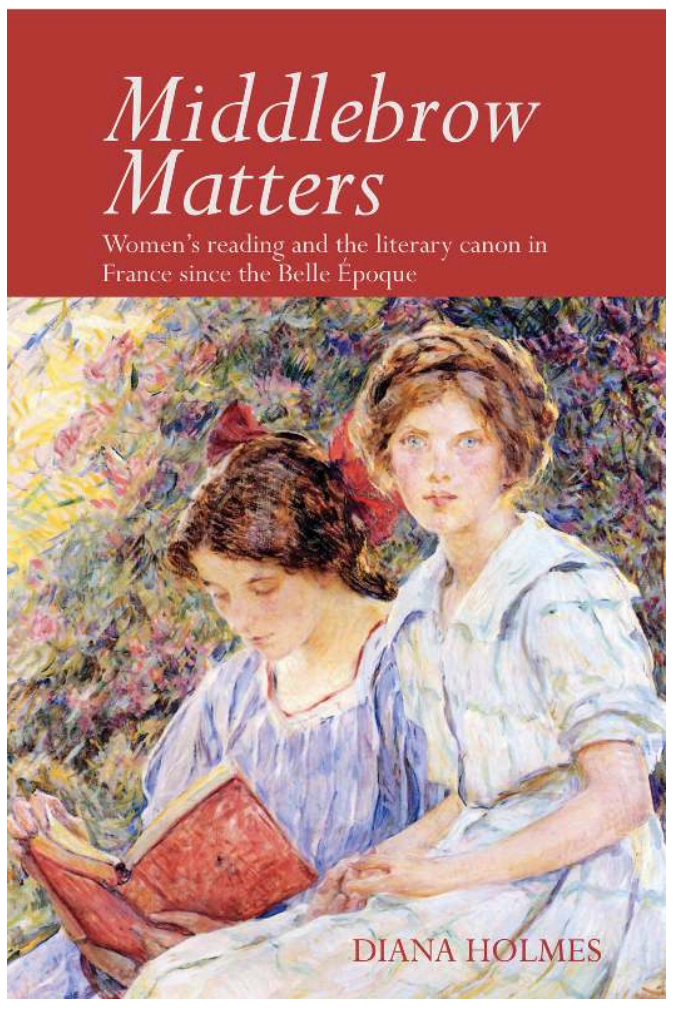

1 Le beau livre de Diana Holmes part d'un double constat. D'abord, du point de vue de la réception, la "littérature moyenne», traduction française assez maladroite de middlebrow, mérite beaucoup mieux que le mépris dans lequel elle est systématiquement maintenue. Car tout comme le highbrow (en français la «production du champ restreint») et le lowbrow (dont la traduction française "littérature populaire » est également minée de cent et un malentendus), le middlebrow fait partie intégrante du système littéraire, sans oublier le fait que le nombre de lecteurs «moyens » dépasse largement, très largement, celui des lecteurs du champ restreint. Ensuite, du point de vue de la production même, la littérature moyenne française continue à être absente du panorama local, à la différence de l'aire anglo-saxonne, où le concept de middlebrow est solidement établi depuis de longues années. Ou si l'on préfère: tout se passe comme si, d'une part, seul existait un discours critique et théorique négligeant le phénomène du middlebrow, et comme si, d'autre part, il n'y avait ni auteurs, ni éditeurs, ni lecteurs ou lectrices proprement français de ce type de littérature.

2 Tout change, heureusement, et c'est aussi le cas de nos rapports avec la culture moyenne. En témoignait déjà le numéro spécial de Bélphégor, dirigé par Diana Holmes et Matthieu Letourneux (vol. 15-2, 2017, https://journals.openedition.org/belphegor/ 921). En témoigne encore davantage la publication de cette monographie importante, qui devrait avoir de grandes répercussions en France.

3 Dans Middlebrow Matters, Holmes rejette une définition purement formelle de ce champ à la fois très spécifique et en partie insaisissable. Son approche combine lecture interne (formelle) et externe (historique, idéologique, sociologique, institutionnelle) de la littérature moyenne. Pour Holmes, le middlebrow vise à offrir une lecture non pas de simple divertissement, mais d'immersion, où l'on se plaît à suivre une intrigue bien faite, à s'identifier à des personnages réalistes et crédibles, à retrouver dans les 
situations et les thèmes du livre les questions de la vie de tous les jours et enfin, fondamentalement, à s'interroger à l'aide d'une fiction, c'est-à-dire par personnages interposés, sur la manière dont il convient de mener sa propre vie. Logiquement, de tels livres, et il s'agit essentiellement de romans, doivent beaucoup de leur succès au contexte dans lequel ils apparaissent et circulent. Le succès du middlebrow dépend en effet de deux conditions au moins: premièrement une offre riche, stable, variée et deuxièmement un public captif, suffisamment lettré et capable d'accéder matériellement aux œuvres et surtout en quête d'une série de réponses que l'état de la société dans lequel on vit n'est pas encore prêt à donner. Il ne faut donc pas s'étonner que le middlebrow soit majoritairement une question de femmes, socialement et juridiquement minorisées mais aussi souvent exclues du champ restreint (solidement tenu par des hommes pour des hommes), et un phénomène britannique, grâce à un système très performant de bibliothèques publiques et un dense réseau d'éditeurs soucieux d'explorer le filon de la production moyenne.

4 Handicapée par l'absence d'un tel système bibliothécaire, du moins dans l'entre-deuxguerres, puis, jusqu'à aujourd'hui, par l'identification quasi absolue entre littérature et littérature de recherche, volontiers définie en termes rigoureusement anti-féminins, notamment par les ukases contre toute forme de sentiment ou d'émotion, la France a toujours été une mauvaise terre d'accueil pour la culture moyenne, en dépit du fait que la très grande majorité de lecteurs de romans sont en fait des lectrices et que les conditions politiques, économiques et idéologiques des femmes françaises ne diffèrent pas radicalement de ce qu'on trouve outre-manche. Certes, la France a eu une politique pro-nataliste plus forte que le Royaume-Uni, elle a aussi été plus lente à accorder le droit de vote, mais les grands débats sur l'accès au marché de l'emploi, l'autodétermination de la femme, les attentes sociales relatives à l'amour, au mariage, à la famille ont été largement comparables.

5 Le sujet du livre de Diana Holmes est triple. Premièrement, Middlebrow Matters offre une excellente synthèse de nos connaissances actuelles sur le sujet, élargies et complétées par de fort utiles recherches francophones (théorie de la fiction de Jean-Marie Schaeffer, théorie de la lecture de Raphaël Baroni, discussions pour ou contre le formalisme lancées par Tzvetan Todorov par exemple). Deuxièmement, Holmes propose aussi une relecture du champ à travers le regard hexagonal, soit hypermasculin et patriarcal, du middlebrow, ce qui aide à mieux comprendre la quasiabsence d'une forme d'écriture qui négocie entre champ restreint et champ élargi. Troisièmement, et on touche là au cœur de la recherche de Holmes, le livre fait aussi le pari, largement tenu d'ailleurs, d'identifier dans le champ français à la fois la présence et l'importance d'un courant littéraire moyen jusqu'ici largement ignoré ou du moins très mal positionné, puisqu'il se trouve soit maladroitement intégré au champ restreint (ce serait le cas de L'Amant de Marguerite Duras), soit relégué sans autre forme de procès aux oubliettes de la littérature populaire et sentimentale (l'œuvre d'Amélie Nothomb en est un bon exemple). Mais comme il n'y a pas de règle sans exception, la littérature française peut se vanter d'avoir Colette, que Diana Holmes relit savoureusement comme représentante d'un middlebrow à la française, c'est-à-dire d'un middlebrow qui n'a pas le droit de dire son nom.

6 L'histoire de la production moyenne en France couvre dans ce livre une période assez large, de la Belle Époque au contemporain. Or même si elle ne se veut nullement exhaustive, la recherche de Diana Holmes arrive à être très équilibrée et finalement 
assez complète. Outre les deux grandes figures que sont Colette et Françoise Sagan, Middlebrow Matters analyse un grand nombre d'autrices et de romans, souvent résumés en grand détail - démarche sans doute moins habituelle mais qui rend souvent de vrais services en montrant l'importance de ce que la théorie littéraire tend à ignorer, à savoir les thèmes, les lieux, le cadre matériel de la fiction (sans oublier le fait que le lecteur académique de Middlebrow Matters risque fort de n'avoir jamais lu, pour les raisons susmentionnées, les livres en question -l'auteur de ce compte rendu plaide volontiers coupable). Mais le livre aborde aussi des éléments plus transversaux comme le système des prix littéraires ou l'action des éditeurs en faveur de la production moyenne.

7 Middlebrow Matters est un livre très novateur, qui devrait avoir un réel impact sur notre façon de penser la littérature, tant du point de vue théorique que du point de vue historique. Une traduction française serait plus qu'utile et pourrait donner lieu à une articulation des travaux sur le middlebrow "féminin », terrain exclusif des recherches de Holmes, avec le middlebrow «masculin ", qui reste largement à définir. De la même manière, les études de la production moyenne pourraient également examiner de plus près les contacts et échanges entre middlebrow et littérature populaire, l'essentiel des efforts s'étant porté jusqu'ici sur les relations complexes entre middlebrow et littérature dite sérieuse. Or dans le cas de la production moyenne, surtout dans le contexte français ou francophone, il est évident que des pistes très prometteuses s'ouvrent à quiconque regarde aussi du côté du roman-photo, mais aussi de celui de la télévision, de la radio, de la culture des magazines, par exemple. Mais chaque chose en son temps : ce qui compte maintenant, c'est de lire Diana Holmes.

\section{AUTHOR}

\section{JAN BAETENS}

Université de Leuven 